\title{
The importance of the anal exam in neurologic classification of spinal cord injury
}

\author{
William H. Donovan ${ }^{1,2}$
}

Received: 20 September 2017 / Revised: 27 September 2017 / Accepted: 27 September 2017

(c) International Spinal Cord Society 2018

\begin{abstract}
The examination of the sensation of the anal orifice and the contraction of the external anal sphincter, either voluntarily or reflexly, has always been an integral part of the International Standards for Neurologic Classification of Spinal Cord Injury (ISNCSCI). Yet the importance of this component has been defended and challenged. This paper compares these two points of view as expressed by Previnaire and Marino, respectively. Both authors make important points but as the papers do not address the same aspect of the anal exam, room for further refinement of ISNCSCI both regarding the details of the exam and the use of components of the exam for prognostication of neurologic recovery is apparent.
\end{abstract}

The points raised in these two presentations illustrate the difficulty in "getting it right the first time" and why ISNCSCI has needed repeated modifications over the years.

In this exercise, for example, even though the purpose of the discussion is to focus on the anal exam, the authors do not address the same aspect of this issue. Previnaire emphasizes the importance of including the Bulbo Cavernosis Reflex (BCR) because of the information it provides regarding the status of the sacral reflex arc and how this portends function of organs of the pelvic viscera. In the acute setting, this information is useful for prognostic purposes although the anal wink and anobulbar reflexes also provide the same information. Even though the bladder, bowel, and sexual functions Previnaire [1] discusses are clearly altered by spinal cord injury (SCI), depending on the status of the sacral reflex arc, the affected organs will eventually declare themselves as to reflexic or areflexic responses even if the BCR is known or not. That however is not justification for not doing it.

I would add that the point he makes about the BCR and thoracic lumbar injuries is useful in distinguishing between epiconal vs both conal and cauda equina injuries as

William H. Donovan

william.donovan@memorialhermann.org

1 The Institute for Rehabilitation and Research (TIRR), TIRR 1333 Moursund St., Houston, TX 77030, USA

2 Department of PM\&R McGovern UT Medical School, Houston, TX, USA
Holdsworth clearly explained [2] and the ISNCSCI booklet and articles illustrate [3, 4]. In the epiconal lesion, the sacral reflex arc may be spared but in the other two it is not spared due to neuron and/or axon destruction.

In contrast to Previnaire, Marino [5] focuses on the relevance of the rectal exam in relation to ISNCSCI and recovery of motor function and points out its vulnerability to unreliability particularly when cognition is impaired. But this is true not just for the examination of the sacral segments [6]. To ask patients whose cognition is faulty or patience is thin to distinguish between normal, impaired, and absent for all dermatomes bilaterally can yield responses that confuse a less experienced examiner.

It is one thing to say that the diagnoses of SCI incompleteness (sensory and/or motor) as Marino opines can be derived in several ways, yet another to say that all of the possible presentations of the ISNCSCI can be recorded consistently and interpreted accurately. The International Standards Committee has attempted over the years insulate ISNCSCI from ambiguity. As Marino points out, one pitfall can occur when someone whose neural function extends below the neurologic level of injury (NLI) but does not extend as far as the sacral segments.

For this reason, the concept of the zone of partial preservation was created to allow for those rare instances where axon conduction can be detected three segments below the NLI but not all the way to the conus such as in the case of a lower second injury. This helped avoid factitious reversal of improvement, i.e., from AIS B to A that was a problem with the Frankel scale as mentioned by Marino. 
The standards committee's decision to require evidence of sacral to cerebral (AIS B) and cerebral to sacral (AIS C and D) conduction respectively before labeling an injury incomplete has been regarded as reasonable over the years and has survived prior modifications of the ISNCSCI because sensation in the external anal sphincter and sharp/ dull sensation around the anal verge as well as voluntary anal contraction of the external sphincter clearly reflects any such conduction to/from the lowest segments of the spinal cord [7].

With regard to Deep Anal Pressure (DAP), previous discussions were held regarding whether DAP alone should qualify for inclusion of the patient as AIS B. If so how it is measured is important as it defies precise dermatomal identification. The conclusion was it was enough to say that if deep pressure was present in the S5 segment, i.e., the external anal sphincter, then at least that sensory modality was able to conduct pass the zone of injury and constituted evidence of AIS B, thus emphasizing the need for consistent performance of the anal examination.

Despite the helpful observations of both authors, reliability is so important that the ground rules, once determined should be adhered to.

The fact that there have been as many revisions of ISNSCI to-date illustrates how hard it is to get things right the first time! The fact that the ISNCSCI is cognition dependent for accurate responses argues for the standards to be kept as least complex as possible. On the other hand if inclusion of the $\mathrm{BC}$ reflex is helpful for prognostication of bladder, bowel, and sexual function whereas the performance of the anal exam is not useful for prognostication of the recovery of motor function, this also should be considered. If assessment of the accuracy of the ISNCSCI shows further discrepancies as recently shown [8], or if imaging and neurophysiologic tools become more available and clinically practical, further refinements of the ISNCSCI may be appropriate.

\section{Compliance with ethical standards}

Conflict of interest The author declares that they have no conflict of interest.

\section{References}

1. Previnaire JG. The importance of the bulbocavernous reflex. Spinal Cord Ser Cases. 2017. https://doi.org/10.1038/s41394-017-0012-0.

2. Holdsworth F. Sir: Fractures, dislocations and fracture - dislocation of the spine. J Bone Jt Surg. 1963;45:6.

3. Holdsworth F. Sir: Fractures, dislocations and fracture - dislocation of the spine. J Bone Jt Surg. 1970;52:1534.

4. Kirshblum S, Biering-Sorensen F, Betz R, Burns S, Donovan W, Graves D, et al. International Standards for Neurological Classification of Spinal Cord Injury; cases with classifications challenges. J Spinal Cord Med. 2014;37:120.

5. Marino RJ. The anorectal exam is unnecessary! Spinal Cord Ser Cases. 2017. https://doi.org/10.1038/s41394-017-0013-z.

6. Kirshblum S, Burns S, Biering-Sorensen F, Donovan W, Graves D, Jha A, et al. International Standards for Neurologic Classification of Spinal Cord Injury (revised 2011). J Spinal Cord Med. 2011;34:535.

7. Oleson C, Burns A, Ditunno J, Geisler F, Coleman W. Prognostic value of pinprick preservation in motor complete, sensory incomplete spinal cord injury. Arch Phys Med Rehabil. 2005;86:988.

8. Alexander M, Aslam H, Marino RJ. Pulse article: How do you do the international standards for neurological classification of SCI anorectal exam? Spinal Cord Ser Cases. 2017;3:17078. 